\title{
Net Fisher information measure versus ionization potential and dipole polarizability in atoms
}

\author{
K. D. Sen ${ }^{1}$, C. P. Panos ${ }^{2}$, K. Ch. Chatzisavvas ${ }^{2 *}$, \\ and Ch. C. Moustakidis ${ }^{2}$ \\ ${ }^{1}$ School of Chemistry, University of Hyderabad, \\ Hyderabad, 500046 India \\ ${ }^{2}$ Department of Theoretical Physics, \\ Aristotle University of Thessaloniki, \\ 54124 Thessaloniki, Greece
}

\begin{abstract}
The net Fisher information measure $I_{T}$, defined as the product of position and momentum Fisher information measures $I_{r}$ and $I_{k}$ and derived from the non-relativistic Hartree-Fock wave functions for atoms with $Z=1-102$, is found to correlate well with the inverse of the experimental ionization potential. Strong direct correlations of $I_{T}$ are also reported for the static dipole polarizability of atoms with $Z=1-88$. The complexity measure, defined as the ratio of the net Onicescu information measure $E_{T}$ and $I_{T}$, exhibits clearly marked regions corresponding to the periodicity of the atomic shell structure. The reported correlations highlight the need for using the net information measures in addition to either the position or momentum space analogues. With reference to the correlation of the experimental properties considered here, the net Fisher information measure is found to be superior than the net Shannon information entropy.
\end{abstract}

Key words: Fisher Information; Information Entropy; Atoms; Ionization potential; Dipole polarizability; Complexity.

\section{Introduction}

Two of the most popular information measures due to Shannon [1 and Fisher [2] respectively, are being increasingly applied in studying the elec-

*e-mail: kchatz@ auth.gr 
tronic structure and properties of atoms and molecules. The Shannon information entropy $S_{r}$ of the electron density $\rho(\mathbf{r})$ in coordinate space is defined as

$$
S_{r}=-\int \rho(\mathbf{r}) \ln \rho(\mathbf{r}) d \mathbf{r},
$$

and the corresponding momentum space entropy $S_{k}$ is given by

$$
S_{k}=-\int n(\mathbf{k}) \ln n(\mathbf{k}) d \mathbf{k},
$$

where $n(\mathbf{k})$ denotes the momentum density. The densities $\rho(\mathbf{r})$ and $n(\mathbf{k})$ are respectively normalized to unity and all quantities are given in atomic units. The Shannon entropy sum $S_{T}=S_{r}+S_{k}$ contains the net information and obeys the well known lower bound by Bialynicki-Birula and Mycielski [3] who obtained the entropic uncertainty relation (EUR) which represents a stronger version of the Heisenberg uncertainty principle of quantum mechanics. Accordingly, the entropy sum in D-dimensions satisfies the inequality [3, 4]

$$
S_{T}=S_{r}+S_{k} \geq D(1+\ln \pi) .
$$

Individual entropies $S_{r}$ and $S_{k}$ depend on the units used to measure $r$ and $k$ respectively, but their sum $S_{T}$ does not i.e. it is invariant to uniform scaling of coordinates.

The Shannon information entropies (uncertainty) provide a global measure of information about the probability distribution in the respective spaces. A more localized distribution in position space corresponds to a smaller value of information entropy. For application of Shannon information entropy in chemical physics we refer the reader to the published literature [6, 7, 8]. An example of quantification of order of the chemical bonding employing Shannon information is $[$.

Analogous applications for other quantum many-body systems (nuclei, atomic clusters and correlated atoms in a trap-bosons) have been reported recently [10].

The Fisher information measure or intrinsic accuracy in position space is defined as

$$
I_{r}=\int \frac{|\nabla \rho(\mathbf{r})|^{2}}{\rho(\mathbf{r})} d \mathbf{r}
$$

and the corresponding momentum space measure is given by

$$
I_{k}=\int \frac{|\nabla n(\mathbf{k})|^{2}}{n(\mathbf{k})} d \mathbf{k}
$$


The individual Fisher measures are bounded through the Cramer-Rao inequality [5] according to $I_{r} \geq \frac{1}{V_{r}}$ and $I_{k} \geq \frac{1}{V_{k}}$, where $V$ 's denote the corresponding spatial and momentum variances respectively. In position space, the Fisher information measures the sharpness of probability density and for a Gaussian distribution is exactly equal to the variance 11. A sharp and strongly localized probability density gives rise to a larger value of Fisher information in the position space. The Fisher measure in this sense is complementary to the Shannon entropy and their reciprocal proportionality is, in fact, utilized in this work. The Fisher measure has the desirable properties, i.e. it is always positive and reflects the localization characteristics of the probability distribution more sensitively than the Shannon information entropy [12. However, for the electronic density distribution in atoms, the enhanced sensitivity of the Fisher measure has not been demonstrated explicitly. The lower bounds of Shannon sum $\left(S_{r}+S_{k}\right)$ and Fisher product $\left(I_{r} I_{k}\right)$ get saturated for the Gaussian distributions. For a variety of applications of the Fisher information measure we refer to the recent book [1] and for applications to the electronic structure of atoms, to the pioneering work of Dehesa et al. [13].

In the context of density functional theory (DFT), Sears, Parr and Dinur 14. were the first to highlight the importance of Fisher information, by showing explicitly that the quantum mechanical kinetic energy is a measure of the information content of a distribution. A link of Shannon information entropy with the kinetic energy for atomic clusters and nuclei has also been indicated in [15]. The electron localization function [16], which has been widely successful in revealing the localization properties of electron density in molecules, has been interpreted in terms of Fisher information [17. Recently, the Euler equation of density functional theory has been derived from the principle of the minimum Fisher information within the time dependent versions [18. The Shannon information sum $S_{T}$ has been used in a large majority of applications of information theory in the electronic structure studies involving atoms and molecules. In this work we define the net information $I_{T}$ as the product $I_{r} I_{k}$ and consider its inverse $I_{T}^{-1}$ as representing the net information similar to $S_{T}$. In this sense we propose to employ $I_{T}$ instead of $S_{T}$ to assess the utility of the net Fisher information vis-a-vis the Shannon entropy sum. This is done in the analysis of experimental properties such as the ionization potential and polarizability, corresponding to the neutral atoms in their ground electronic states. It is worth noting here that the net uncertainty measures defined in the conjugate spaces are at the foundation of the quantum mechanical probability distribution. As noted 
above, the quantities $I_{T}$ and $S_{T}$ measure the net information content of the probability distribution including its spatial characteristics. Such measures could therefore be tested in their ability to reproduce the trends in atomic sizes, ionization potentials and the polarizabilities, respectively.

Very recently the question whether atoms can grow in complexity with the increase in nuclear charge has been addressed 20, 21. In particular the Onicescu information measure 22 in position space $E_{r}$ and momentum $E_{k}$ have been defined as the corresponding density expectation values $E_{r}=$ $\int \rho(\mathbf{r})^{2}(\mathbf{r})=\langle\rho(\mathbf{r})\rangle$ and $E_{k}=\int n(\mathbf{k})^{2} d(\mathbf{k})=\langle n(\mathbf{k})\rangle$, respectively.

The complexity $C$ is measured accordingly to the prescription due to Lopez-Ruiz, Manchini and Calbet (LMC) [23, 21] as

$$
C=S_{T} E_{T},
$$

where $E_{T}=E_{r} E_{k}$.

$S_{T}$ denotes the information content stored in the system and $E_{T}$ corresponds to the disequilibrium of the system, i.e. the distance from its actual state to equilibrium, according to 23. Shiner, Davison and Landsberg (SDL) 24] and LMC measures were criticized in [25, 26, 27]. A related discussion can be found in [20, 21].

In the light of its sensitivity to describe the localization property it is useful to consider in the above equation $I_{T}^{-1}$ instead of $S_{T}$, to define LMC complexity measure based on the net Fisher information. Thus a new definition of complexity measure (LCM-like) is the following

$$
C=E_{T} I_{T}^{-1}
$$

It is generally agreed that the ionization potential (I.P.) and static dipole polarizability $\alpha_{d}$ represent the two key electronic properties of atoms and molecules, which control a host of their other properties including chemical reactivity. Indeed, the DFT descriptions of chemical reactivity [28] such as electronegativity, hardness and Fukui functions are intimately related to I.P. and $\alpha_{d}$. Due to this reason several interesting studies have been made earlier [29, 30] to find a correlation between the two experimental properties. The purpose of this paper is to examine how well the net Fisher information measure correlates with the experimental values of the inverse of the first I.P. and $\alpha_{d}$, of neutral atoms. In particular, we shall be interested in the relative advantages of using the net Fisher information over the Shannon information entropy sum. In a similar manner we also report here the LMC complexity measure based on $I_{T}^{-1}$ and point out some of the new features which are not displayed when the $S_{T}$ is used instead [21]. 


\section{Results}

\subsection{Ionization potentials and $I_{T}$}

Throughout this work we have used the consistent data on the spherically averaged density 31] derived from the highly accurate analytic Hartree-Fock (HF) wave functions 32. These results are in quantitative agreement with our results reported earlier, i.e. those derived form the analytic HF wave functions due to Bunge et al 33. In a large number of cases, we have also computed the information measures using numerical HF wave functions and have found similar quantitative agreement. We note here that the present results do not include any electron correlation and/or relativistic effects. The experimental ionization potential of neutral atoms has been taken from reference 34].

In Fig. 1 we plot $S_{T}$ and I.P. as functions of $Z$. It is observed that $S_{T}$ does not perform as a sensitive information measure reproducing the details of the trends in I.P. It does show the gross atomic periodicity in terms of the shell structure as the humps.

In Fig. 2 the values of $I_{T}$ (instead of $S_{T}$ as in Fig. 1] and the inverse of I.P. are plotted as functions of $Z$. Compared to Fig. 1 the two curves in Fig. 2, resemble each other in far more details. It has been shown earlier that $I_{k}$ behaves similarly to I.P. for atoms 31. The net Fisher information amplifies the details of correlation by approximately two orders of magnitude. Our aim of plotting $I_{T}$ versus inverse of I.P. is to show the similarities in the two curves in the upward direction and also lay emphasis on the net Fisher information, $I_{T}$. We note here that in subsection 2.3 it is

$I_{T}^{-1}$ that enters the definition of complexity which directly correlates with I.P. Very recently, the idea of taking the relative Shannon entropy of an element within a group of the periodic table with respect to the inert gas atom, located at the end of the group has been proposed 35] in order to get a more sensitive quantum similarity measure of density distributions. It would be interesting to investigate whether the correlations found in Fig. (2) can be further improved by using such a similarity measure for each group using the Fisher information according to

$$
\Omega(Z)=1-\left[\frac{I_{T(r e f)}}{I_{T(Z)}}\right]
$$

where $\Omega(Z)$ measures the distance in compactness of the element $Z$ from the most compact ideal gas atom in the same group, used as reference. A larger value of $\Omega(Z)$ would correspond to smaller I.P. Our use of $\Omega(Z)$ is 
inspired by Landsberg's definition of order parameter $\Omega: \Omega=1-\Delta$, where

$\Delta=\frac{S_{T}}{S_{\max }}$ is a disorder parameter given in terms of the actual entropy $S$ and the maximum possible entropy $S_{\max }$ of the system 36. An application of $\Omega$ in quantum many-body systems was carried out in [37.

In Fig. 3 we present $\Omega$ and [I.P. $]^{-1}$ as functions of $Z$. It is found that the correlation is more direct than that obtained in Fig. 2. This observation suggests that $\Omega(Z)$ can be used as a measure of quantum similarity of atoms and opens up a new application of the net Fisher information measure $I_{T}$.

\subsection{Dipole polarizability and $I_{T}$}

The variation of polarizability $\alpha_{d}$ of atoms with $S_{T}$ is found to be essentially similar to that of $S_{T}$ versus I.P., as already given in Fig. 1. In the background of such insensitivity of $S_{T}$, we shall now consider the correlation of $I_{T}$ with the experimental estimates of polarizability $\alpha_{d}$. The experimental values have been taken from the compilation of Miller and Bederson 38, for atoms with $Z=1-88$. The variation of $I_{T}$ and $\alpha_{d}$ with $Z$ for atoms with $Z=1-88$ is shown in Fig. 4. The overall correlation is found to be excellent with the maximum polarizability elements of the alkali atoms immediately following the sharply increasing values if $I_{T}$ just after the inert gas atoms. The polarizability predicted by $I_{T}$ for the alkaline atoms present themselves as the only examples which are not sufficiently well discriminated against the neighboring atoms, in this case, the alkali metal atoms. It appears that the compactness described by $I_{T}$ in going from the valence electron configuration of $(n s)^{1}$ to $(n s)^{2}$ does not increase sharply enough to quantitatively reflect the changes in polarizability from alkali to alkaline earth atoms. For these examples it is advisable to carry out further computations of the Fisher information using wave functions which include electron correlation effects.

\subsection{Complexity using $I_{T}$}

Finally, we discuss the LMC measure of complexity using Eq.(6) in which $S_{T}$ is substituted with $I_{T}^{-1}$. In an earlier publication [20], the SDL measure of complexity (for various indices of disorder and order) has been plotted as a function of $Z$ and a series of oscillations around a certain average value, was obtained. This led to the conclusion that atoms cannot grow in complexity as $Z$ increases. The latter conclusion was modified in 21, where a similarity of SDL (for magnitude of disorder equal to zero and magnitude of order equal to four) and LMC measures led to the observation that $C$ is an increasing function of $Z$. Here, in Fig. [5, we present the measure $C=E_{T} I_{T}^{-1}$ as 
a function of $Z$. This curve points to a gradual decrease in complexity with systematic oscillations due to new shells added on as $Z$ increases. It is seen that while the fluctuations of complexity following the periodicity of the elements represent a general feature, the issue of the behavior of complexity (increase or decrease) with increasing $Z$, cannot be answered at present with certainty. Such an answer, would be desirable towards the proper description of organization of quantum systems. A separate plot of $E_{T}$ and $I_{T}^{-1}$ in Fig. [6] suggests that the trend shown in Fig. 5 is controlled by $I_{T}^{-1}$. While the earlier conclusion about the oscillations of complexity in atoms stands vindicated, the use of Fisher information measure leads to a more transparent variation of $C$ as a function of $Z$. It is also indicated that the decreasing complexity with increasing $Z$ is bounded by the successive shells as oscillations.

\section{Summary}

In conclusion, we have found that the net Fisher information denoted by the product of the Fisher information in position and momentum spaces (Eq.(4)-(5D) ) describes the variation of the I.P. and the static dipole polarizability as a function of $Z$, more efficiently than the Shannon information entropy sum (Eq.(11)-(2)). Our results also highlight the importance of using the net information in addition to those corresponding to either the position or momentum space separately, in order to analyze and predict the experimental properties. Furthermore, the LMC measure of complexity of atoms as a function of $Z$ is found that can be transparently expressed employing the net Fisher information. It would be interesting to extend the application of net Fisher information entropy to nuclear, molecular and atomic cluster densities.

\section{Acknowledgements}

K.D. Sen acknowledges with thanks the Department of Theoretical Physics, Aristotle University of Thessaloniki, for its warm hospitality. He is also grateful to Prof. Antoniou Ioannis for constant encouragement and to Professor E. Romera for data sharing. The work of K.D. Sen, C.P. Panos and K.Ch. Chatzisavvas, was supported by Herakleitos Research Scholarships (21866) of EПЕАЕK and the European Union. 


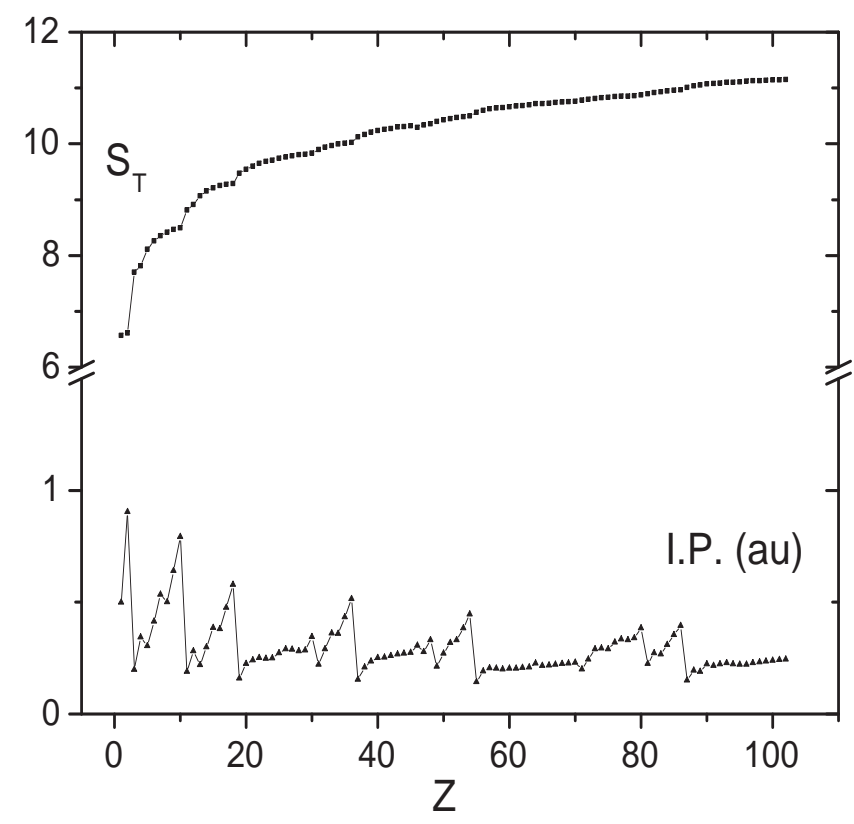

Figure 1: $S_{T}$ and I.P. as functions of $Z$ 


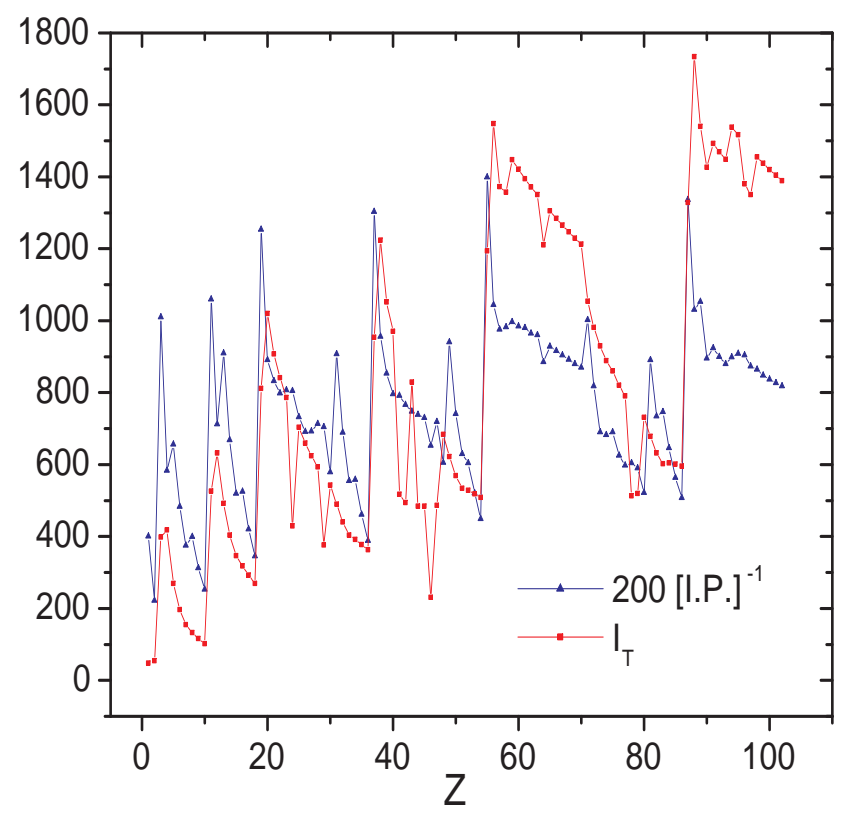

Figure 2: $I_{T}$ and I.P. as functions of $Z$ 


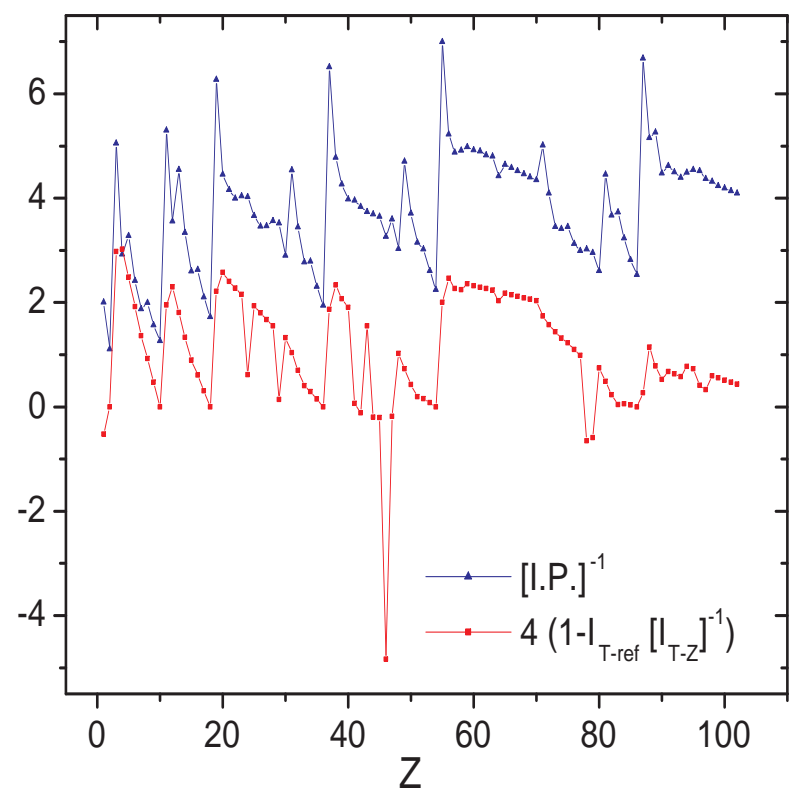

Figure 3: $\Omega(Z)$ and [I.P. $]^{-1}$ as functions of $Z$ 


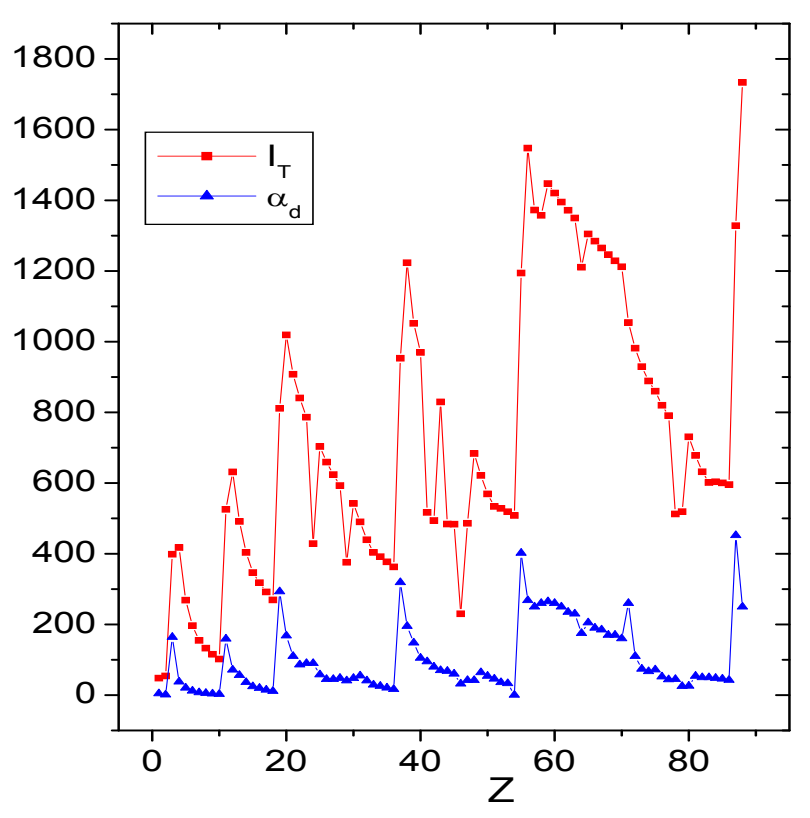

Figure 4: $I_{T}$ and $\alpha_{d}$ as functions of $Z$ 


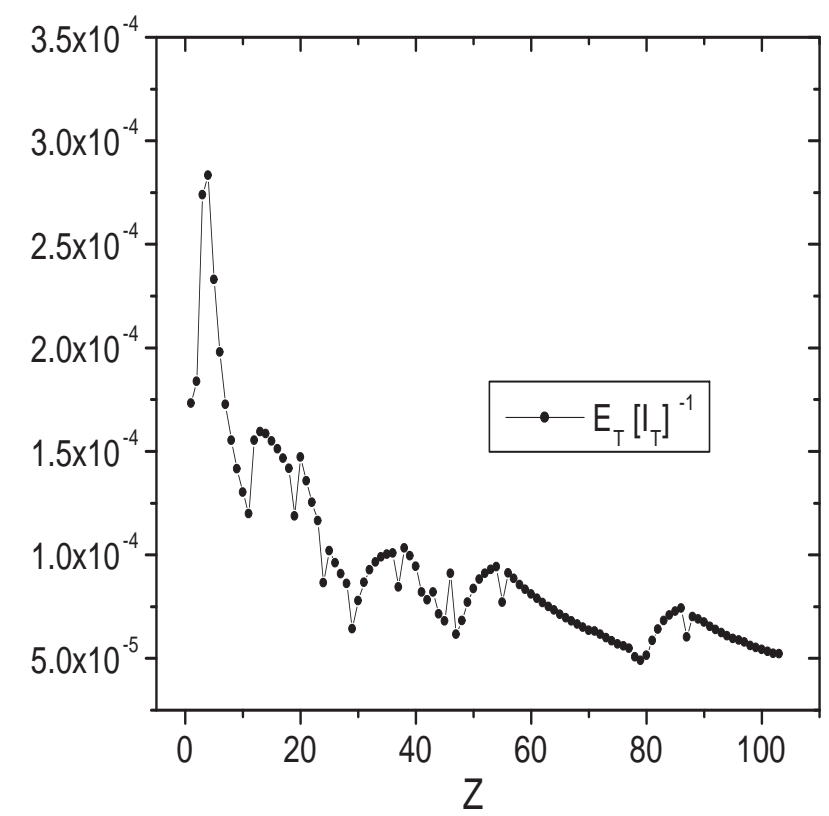

Figure 5: $C=E_{T} I_{T}^{-1}$ as a function of $Z$ 


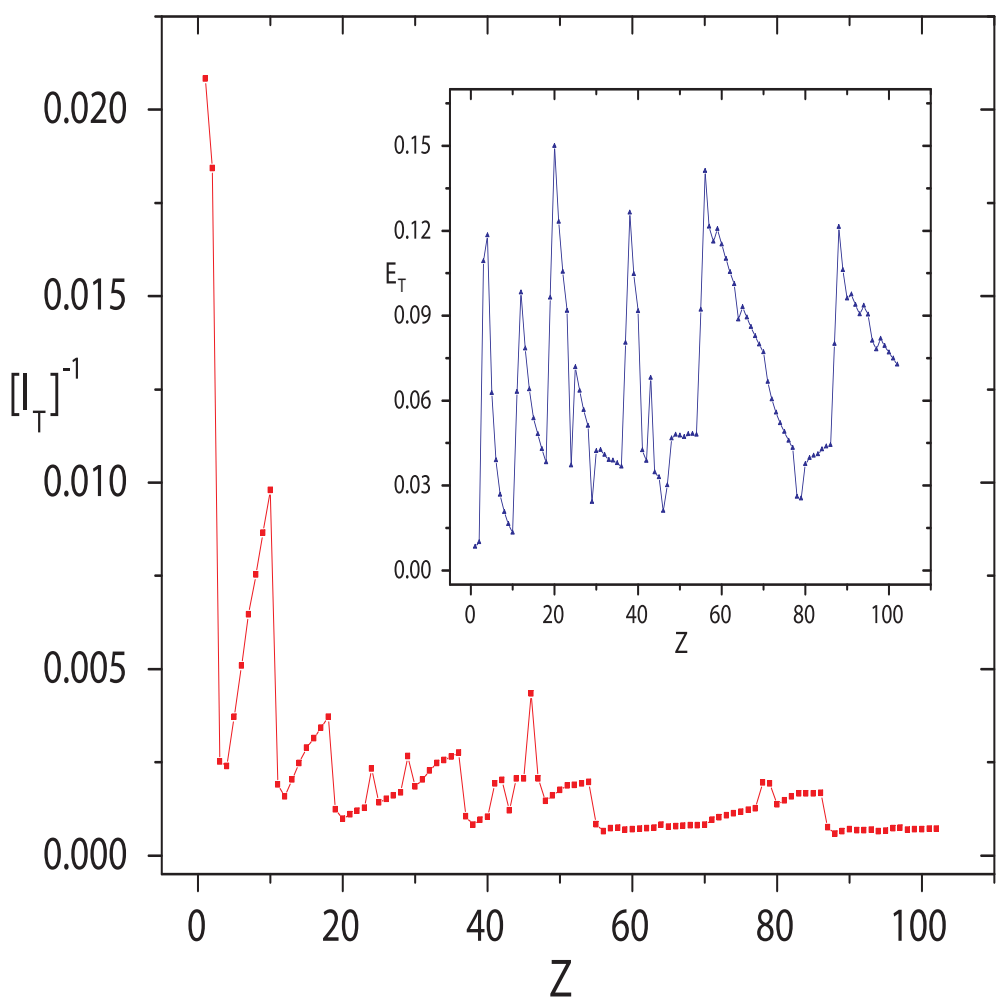

Figure 6: $E_{T}$ and $I_{T}^{-1}$ as functions of $Z$ 


\section{References}

[1] C.E. Shannon, Bell Syst. Tech. 27, 379 (1948); ibid. 27, 623 (1948).

[2] R.A. Fisher, Proc. Cambridge Phil. Sec. 22, 700 (1925).

[3] I. Bialynicki-Birula, J. Mycielski, Commun. Math. Phys. 44, 129 (1975).

[4] S.B. Sears, Ph.D. thesis, Applications of Information Theory in Chemical Physics, University of North Carolina at Chapel Hill (1980).

[5] C.R. Rao, Linear Statistical Interference and its Applications, (Wiley, New York, 1965); A. Stam, Inf. Control 2, 101 (1959).

[6] S.R. Gadre, S.B. Sears, S.J. Chakravorty, and R.D. Bendale, Phys. Rev. A 32, 2602 (1985); S.R. Gadre, Phys. Rev. A 30, 620 (1984); S.R. Gadre and R.D. Bendale, Int. J. Quant. Chem. 28, 311 (1985); S.R. Gadre, S.A. Kulkarni, and I.H. Shrivastava, Chem. Phys. Lett. 16, 445 (1990); S.R. Gadre, R.D. Bendale, and S.P. Gejji, Chem. Phys. Lett. 117, 138 (1985); S.R. Gadre and R.D. Bendale, Curr. Sci. (India) 54, 970 (1985); S.R. Gadre, Reviews of Modern Quantum Chemistry, (edited by K.D. Sen, World Scientific, Singapore 2002) p.108.

[7] A.N. Tripathi, V.H. Smith, Jr., R.P. Sagar, and R.O. Esquivel, Phys. Rev. A 54, 1877 (1996); M. Ho, D.F. Weaver, V.H. Smith, Jr., R.P. Sagar, and R.O. Esquivel, Phys.Rev. A 57, 4512 (1998); M. Ho, V.H. Smith, Jr., D.F. Weaver, C. Gatti, R.P. Sagar, and R.O. Esquivel, J. Chem. Phys. 108, 5469 (1998); J.C. Ramirez, J.M.H. Perez, R.P. Sagar, R.O. Esquivel, M. Ho, and V.H. Smith, Jr., Phys. Rev. A 58 3507 (1998); M. Ho, D.F. Weaver, V.H. Smith, Jr., R.P. Sagar, R.O. Esquivel, and S. Yamamoto, J. Chem. Phys. 109, 10620 1998; R.P. Sagar, J.C. Ramirez, R.O. Esquivel, M. Ho, and V.H. Smith, Jr., Phys. Rev. A 63, 022509 (2001); N.L. Guevara, R.P. Sagar, and R.O. Esquivel, J. Chem. Phys. 119, 7030 (2003); ibid 122, 084101 (2005); Q. Shi and S. Kais, J. Chem. Phys. 121,5611(2004); ibid 309127 (2005).

[8] R.J. Yanez, W. Van Assche, and J.S. Dehesa, Phys. Rev. A 50, 3065 (1994).

[9] P. Karafiloglou and C.P. Panos, Chem. Phys. Lett. 389, 400 (2004).

[10] C.P. Panos and S.E. Massen, Int. J. Mod. Phys. E 6, 497 (1997); S.E. Massen, Ch.C. Moustakidis, and C.P. Panos, Phys. Lett. A 64, 131 
(2002); S.E. Massen and C.P. Panos, Phys. Lett. A 246, 530 (1998); S.E. Massen and C.P. Panos, Phys. Lett. A 280, 65 (2001); G.A. Lalazissis, S.E. Massen, C.P. Panos, and S.S. Dimitrova, Int. J. Mod. Phys. E 7, 485 (1998); K.Ch. Chatzisavvas, S.E. Massen, Ch.C. Moustakidis and C.P. Panos, Intl. J. Mod. Phys. B 20, 2189 (2006).

[11] B.R. Frieden, Science from Fisher Information, (Cambridge University Press, 2004).

[12] R.W. Carroll, Fluctuations, Information, Gravity and Quantum Potential (Springer, Dordrecht 2006), p.240; P. Garbacjewski, J. Stat. Phys. 123, 315 (2006), p.317, p.328.

[13] J.S. Dehesa, A. Martinez-Finkelstein, and J. Sanchez-Ruiz, J. Comp. Appl. Math. 133, 23 (2001); E. Romera, P. Sanchez-Moreno, and J.S. Dehesa, Chem. Phys. Lett. 414, 468 (2005); E. Romera and J.S. Dehesa, J. Chem. Phys. 120, 8906 (2004); J.S. Dehesa, A. Martinez-Finkelstein, and V.N. Sorokin, Mol. Phys. 104, 613 (2006); J.S. Dehesa, S. LopezRosa, B. Olmos, and R.J. Yanez, J. Math. Phys. 47, 052104 (2006); J.S. Dehesa, B. Olmos, and R.J. Yanez, ArXiv:math.CA/0606133 v1 (2006).

[14] S.B. Sears, R.G. Parr, and U. Dinur, Israel Journal of Chem. 19, 165 (1980).

[15] S.E. Massen and C.P. Panos, Phys. Lett. A 280, 65 (2001).

[16] A.D. Becke and K.E. Edgecombe, J. Chem. Phys. 92, 5397 (1990).

[17] R.F. Nalewajski, A.M. Koester, and S. Escalante, J. Phys. Chem. A 109, 10038 (2005).

[18] A. Nagy, J. Chem. Phys. 119, 9401 (2003).

[19] K.D. Sen, J. Chem. Phys. 123, 074110 (2005); N.L. Guevara, R.P. Sagar, and R.O. Esquivel, Phys. Rev. A 67, 012507 (2003).

[20] K.Ch. Chatzisavvas, Ch.C. Moustakidis, and C.P. Panos, J. Chem. Phys. 123, 174111 (2005).

[21] C.P. Panos, K.Ch. Chatzisavvas, Ch.C. Moustakidis, and E.G. Kyrkou ArXiv:quant-ph/0605082 v2 (accepted for publication in Phys. Lett. A) (2006). 
[22] O. Onicescu, CR Acad. Sci. Paris A 263, 25 (1966).

[23] R. Lopez-Ruiz, H. Manchini, and X. Calbet, Phys. Lett. A 209, 321 (1995).

[24] J.S. Shiner, M. Davison, and P.T. Landsberg, Phys. Rev. E 59, 1459 (1999).

[25] J. Crutchfield, D.P. Feldman, and C.R. Shalizi, Phys. Rev. E 62, 2996 (2000).

[26] D.P. Feldman and J. Crutchfield, Phys. Lett. A 238, 244 (1998).

[27] R. Stoop, N. Stoop, A. Kern, and W-H Steeb, J. Stat. Mech.: Theory and Experiment, 11009 (2005).

[28] P. Geerlings, F. De Proft, and W. Langnekar, Chem. Rev. 103, 1793 (2003).

[29] B. Fricke, J. Chem. Phys. 84, 862 (1986).

[30] P. Politzer, P. Jin, and J.S. Murray, J. Chem. Phys. 117, 8197 (2002).

[31] E. Romera, Mol. Phys. 100, 3325 (2002).

[32] T. Koga, K. Kanayama, S. Watanabe, T. Imai, and A.J. Thakkar, Chem. Acc. 104, 411 (2002).

[33] C.F. Bunge, J.A. Barrientos, and A.V. Bunge, At. Data Nucl. Data Tables 53, 113 (1993).

[34] R.G. Parr, L.J. Bartolotti, J. Am. Chem. Soc. 104, 3801 (1982).

[35] A. Borgoo, M. Godfroid, K.D. Sen, F. De Proft, and P. Greerlings, Chem. Phys. Lett. 399, 363 (2004).

[36] P.T. Landsberg, Phys. Lett. A 102, 171 (1984).

[37] C.P. Panos, Phys. Lett. A 289, 287 (2001).

[38] T.H. Miller and B. Bederson, Adv. At. Mol. Phys. 13, 1 (1977). 